\title{
Diagnostic value and clinical application of next-generation sequencing for infections in immunosuppressed patients with corticosteroid therapy
}

\author{
Sen Wang ${ }^{1 \#}$, Jingwen $\mathrm{Ai}^{1 \#}$, Peng Cui ${ }^{1}$, Yimin $\mathrm{Zhu}^{1}$, Honglong $\mathrm{Wu}^{2,3}$, Wenhong Zhang ${ }^{1}$ \\ ${ }^{1}$ Department of infectious disease, Huashan Hospital of Fudan University, Shanghai 200040, China; ${ }^{2}$ Binhai Genomics Institute, Tianjin \\ Translational Genomics Center, BGI-Tianjin, Tianjin 300308, China; ${ }^{3}$ BGI Genomics, BGI-Shenzhen, Shenzhen 518083, China \\ Contributions: (I) Conception and design: S Wang, J Ai, W Zhang; (II) Administrative support: H Wu, W Zhang; (III) Provision of study materials or \\ patients: S Wang, P Cui, Y Zhu; (IV) Collection and assembly of data: J Ai, P Cui, Y Zhu; (V) Data analysis and interpretation: S Wang, J Ai, H Wu; \\ (VI) Manuscript writing: All authors; (VII) Final approval of manuscript: All authors. \\ \#These authors contributed equally to this work. \\ Correspondence to: Wenhong Zhang. Department of Infectious Diseases, Huashan hospital of Fudan University, 12 Wulumuqi Zhong Road, Shanghai \\ 200040, China. Email: zhangwenhongfd@126.com.
}

\begin{abstract}
Background: Next-generation sequencing (NGS) is a comprehensive approach for sequence-based identification of pathogens. However, reports on the use of NGS in patients with immunosuppression are scarce, especially in subjects with negative microbiological results.

Methods: In this study, NGS was performed on samples obtained from 108 anonymized patients with suspected infection undergoing immunosuppressive corticosteroid therapy. A panel of conventional microbiological tests (CMT) was performed in parallel with NGS.

Results: Of these 108 subjects, 36 were diagnosed with infections by clinical and microbiological criteria (Group I), 41 were exclusively diagnosed clinically (Group II), and 31 exhibited no evidence of infection (Group III). In Group I, NGS was concordant with CMT results from 29 patients (80.6\%). A total of 4 samples had positive NGS results in Group III. NGS showed a sensitivity of $80.6 \%$ (95\% CI, 64.7\% to $90.6 \%$ ) and specificity of $87.1 \%$ (95\% CI, $70.5 \%$ to $95.5 \%$ ). NGS also played an important role in optimizing antibiotic regimens in patients with negative results for CMT (Group II). The treatment success rate (TSR) of patients using NGS-guided antibiotic regimens $(81.8 \%, 18 / 22)$ was significantly higher than that of patients using empirical antibiotics $(52.6 \%, 10 / 19)(\mathrm{P}<0.0001)$. NGS results were not affected by the degree of immunosuppression.

Conclusions: NGS of clinical samples from immunosuppressed patients demonstrated promising diagnostic potential in identifying clinically relevant pathogens. Consequently NGS stands to become a standard tool for infection detection and control, providing valuable information to optimize antibiotic therapy in immunosuppressed patients.
\end{abstract}

Keywords: Next-generation sequencing (NGS); infection; diagnosis; polymicrobial infection; immunodeficiency

Submitted Oct 06, 2019. Accepted for publication Dec 24, 2019.

doi: 10.21037/atm.2020.01.30

View this article at: http://dx.doi.org/10.21037/atm.2020.01.30

\section{Introduction}

Immunocompromised hosts have an increased susceptibility to infections for a wide variety of reasons, the primary of which is the compromised status of immune system components that contribute to host defense against infection. Including the integrated system of physical barriers as well as innate and adaptive immunity (1). In clinical settings, secondary immunodeficiency diseases are more common 
than primary ones, as the former develop mostly as the result of a specific condition that is treatment-related. The most common of these treatments is the therapeutic application of glucocorticoids for a variety of diseases due to their anti-inflammatory and immunosuppressive properties (2). Glucocorticoid use increases the risk of systemic infection in a dose-dependent manner (3).

After infection in immunosuppressed patients, both inaccurate and insufficient therapeutic coverage can lead to increased risk of side effects and decreased quality of life (3); therefore, timely and accurate microbial identification is critical. Smear microscopy and culture are still the most commonly used microbiological methods to identify pathogens in clinical settings; however, both methods are relatively insensitive and pathogen culture is timeconsuming $(4,5)$. Histopathological diagnosis is the gold standard for several kinds of infection including invasive fungal infections. However, it also requires substantial time and is not pathogen-specific. Other diagnostic methods including nucleic acid amplification tests (NAAT), serologic tests, and immunological diagnostic tests have yielded potential diagnostic value. However, these methods are either invasive or restricted to detecting a limited number of suspected microorganisms $(6,7)$. Therefore, rapid microbiological diagnosis of infections is urgently needed to facilitate the timely application of antimicrobial therapy.

In recent decades, next-generation sequencing (NGS) has been improving constantly, entering clinical practice to provide a powerful tool applied across medical settings with spectacular success $(4,8)$. Compared to culture-based methods, NGS offers advantages including short turnaround-time and unbiased quantitative or semi-quantitative analysis (9-13). In addition to these specific benefits, multiple agents across the full microbial spectrum contributing to disease can be simultaneously detected by NGS, as along with the identification of nonculturable microbes (8). Recent work has highlighted the current interest in using NGS for the identification of pathogens in the diagnosis of several kinds of diseases including respiratory $(13,14)$, urinary tract $(15)$, central nervous system (CNS) (16), bloodstream (17), and periprosthetic joint infections (12). However, reports on the use of NGS in immunosuppressed hosts remain scarce-especially in subjects with negative results from conventional microbiological methods. Clinical experience with application of NGS is still relatively limited. Due to the unique characteristics and overall severity of infections in immunosuppressed patients, the evaluation of rapid and accurate diagnostic methods is of the utmost urgency. This study aims to determine whether NGS technology can meet this need, by assessing its ability to detect pathogens in febrile immunosuppressed patients and examining its correspondence with conventional clinical microbiological tests.

\section{Methods}

\section{Study design}

In this study, we prospectively recruited immunosuppressed patients with suspected infections admitted to Huashan Hospital in Shanghai, China. Subjects were enrolled sequentially between March 25, 2017, and April 14, 2018, within the first $72 \mathrm{~h}$ of a suspected infection. All of the immunocompromised subjects received corticosteroids as treatment for rheumatic or autoimmune diseases, drug allergies, etc. (Table 1). Infections were suspected in these patients due to symptoms including fever, cough, weakness, and flushed appearance. The enrollment criteria are listed in Table S1; 9 patients had been exclude due to previous evidence of pathogenic infection (Figure 1). An ethical review application was validated by the Ethical Review Committee of Huashan Hospital, Fudan University, Shanghai, China. Written informed consent was obtained for each patient or their legal designees prior to participation.

\section{Specimen collection and processing}

During enrollment, all of the subjects received conventional microbiological tests (CMT) ordered by their treating clinicians. Different clinical specimens were collected for testing based on the type of suspected infection (i.e., blood samples, sputum, nasopharyngeal swabs, puncture fluids, bronchoalveolar lavage fluid, and aspirates). The CMT used in this study are presented in Table S1; they included blood culture, serological tests, molecular diagnostic tests, antigen detection, and direct examination of specimens. The CMT were conducted according to clinically assessed necessity. During this time, additional samples were collected and shipped overnight to Huada Laboratories (Shenzhen, China) for NGS.

\section{Case definitions}

All patients in this study received individualized treatment and were followed until the end of treatment. All clinical data and laboratory and radiographic test results were 
Table 1 Demographics and clinical characteristics of the enrolled immunosuppressed subjects with suspected infection

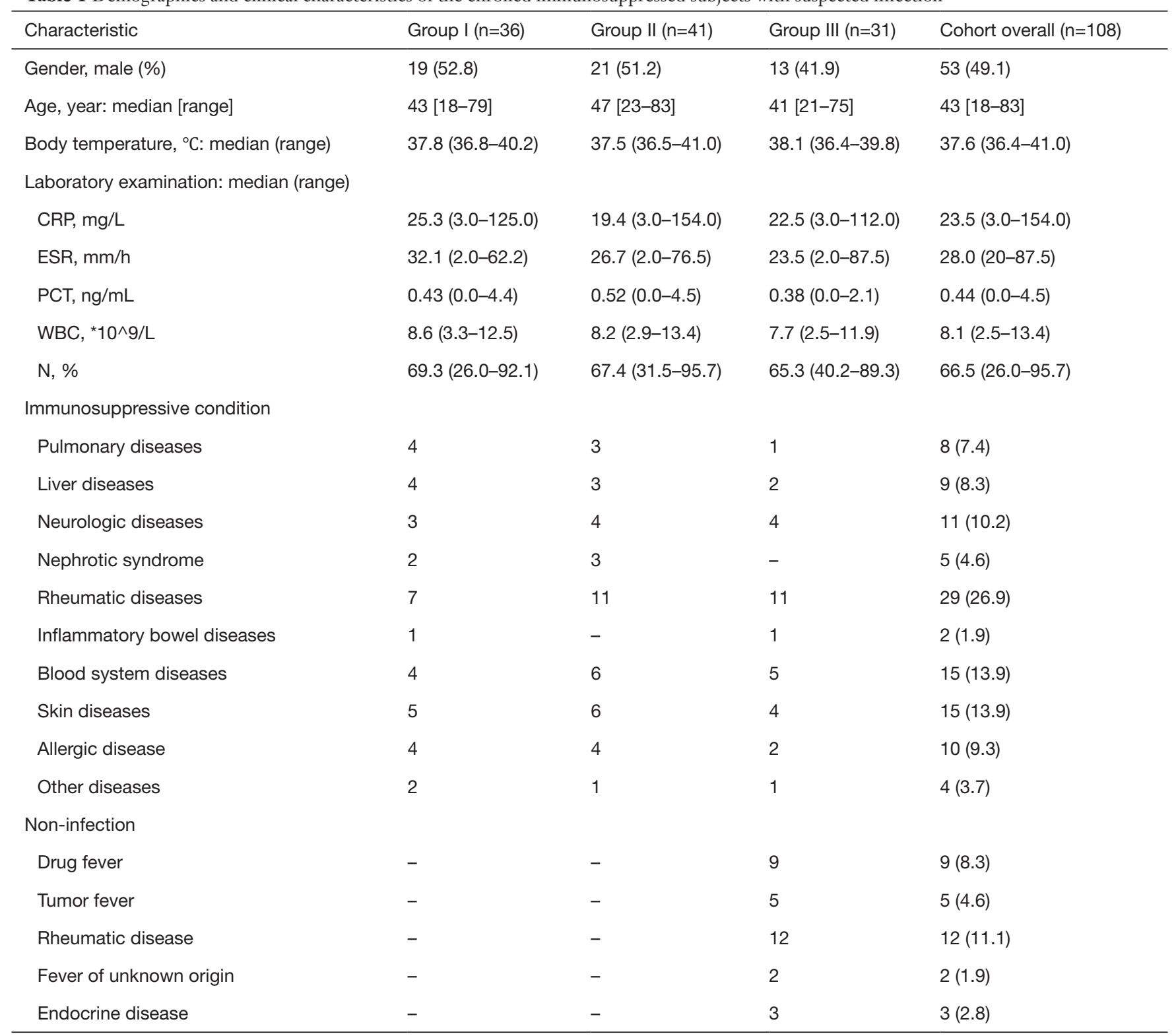

Group I: subjects who met both clinical and microbiologic criteria for infection. Group II: subjects who met clinical criteria for infection only. Group III: subjects with noninfectious etiology. CRP, C-reaction protein; ESR, erythrocyte sedimentation rate; PCT, procalcitonin; WBC, white blood cell; N, neutrophil.

recorded. Diagnoses were made by two independent clinicians blinded to the NGS results who retrospectively reviewed each patient's medical record including clinical, microbiological, and treatment outcome information to determine whether they met the case definitions of different kinds of infection, with respect to microbiologic and/or clinical criteria (Table S1). Using this approach, the patients were subdivided into three groups: group I was defined by both clinical and laboratory criteria; group II was defined by clinical criteria only; and group III included patients with no evidence of infection who's standard CMT results were negative.

\section{Sample processing and library construction}

Nucleic acids (DNA and RNA) were extracted from 


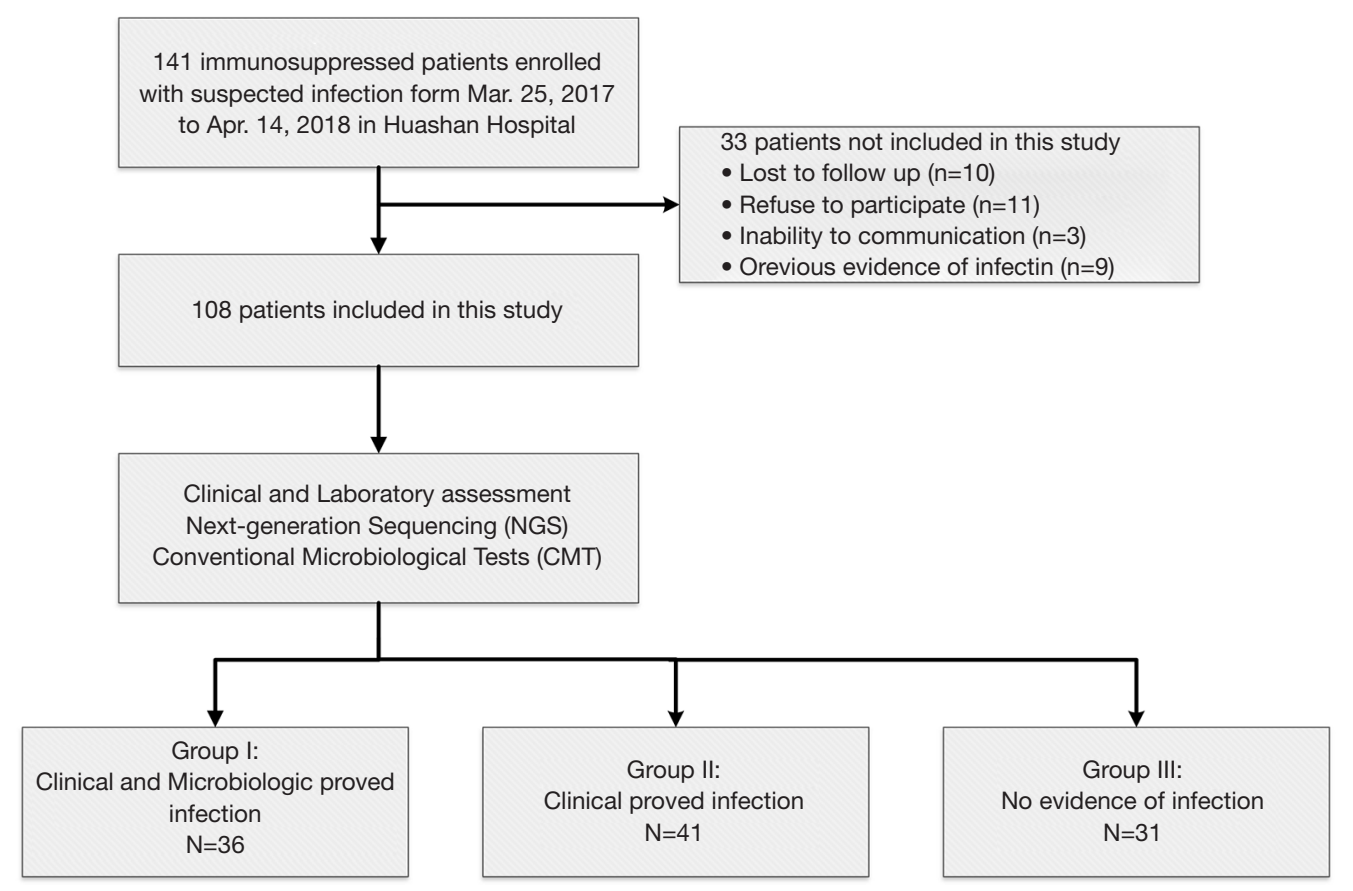

Figure 1 Trial profile.

collected samples according to standard procedures. A 300 $\mathrm{uL}$ sample of plasma samples and other biological fluids were collected in Dnase/Rnase tubes for the identification of potential pathogens. A common issue in metagenomic sequencing is the introduction of contaminating microbial nucleic acid during sample preparation. The potential contaminating sources include PCR reagents, nucleic acid extraction kits, human skin, as well as environment. In order to control the effect of contamination, a negative control was prepared in parallel and sequenced in the same run. Before nucleic acid extraction, samples were processed as follows: the tissue sample was grinded into homogenate, the urine was concentrated and the sputum was liquefied.

DNA and total RNA was extracted with TIANamp Micro DNA Kit (DP316, TIANGEN BIOTECH, Beijing, China) and QIAamp Viral RNA Mini Kit (52906, Qiagen, China) following the manufacturer's operational manual, respectively. The RNA was reverse transcribed and synthesized to double-stranded complementary DNA (ds cDNA) with SuperScript II Reverse Transcription Kit (18064-014, Invitrogen, China). In addition to Plasma, the DNA/cDNA from other sample was fragmented using Bioruptor Pico instrument to generate 200-300 bp fragments (Bioruptor Pico protocols). Then, the libraries were constructed as follows: first, the DNA fragments were subjected to end- repair and add A-tailing in one tube, subsequently, the resulted DNA was ligated with bubbleadapters which contained barcode sequence, and then amplified by PCR method. Quality control was carried out using bioanalyzer (Agilent 2100, Agilent Technologies, Santa Clara, CA, USA) to assess the DNA concentration and fragment size. Qualified libraries were pooled together to make single strand DNA circle (ssDNA circle) and then generated DNA nanoballs (DNBs) by rolling circle replication (RCA). The final DNBs were loaded into sequence chip and sequenced on BGISEQ platform using $50 \mathrm{bp} /$ single-end sequencing. Finally, the optical signals were collected using high resolution imaging system, and then the optical signals were transformed into digital information, which can be decode into DNA sequence information.

\section{Bioinformatics pipeline}

All raw reads were quality filtered using made-in-house program, including filtering adapter contamination, low quality and low-complexity reads. Next, the clean reads after quality filtering were mapped to a human reference database including hg38 and Yanhuang genome sequence using Burrows-Wheeler Alignment (Version: 0.7.10). 
Remained reads were aligned to the nonredundant bacterial, virus, fungal, and parasite databases using BurrowsWheeler Alignment (Version: 0.7.10). The mapped data were processed for advanced data analysis. We downloaded all the reference genome from the public database, such as NCBI (ftp://ftp.ncbi.nlm.nih.gov/genomes/). Currently, our databases contain 4,152 whole genome sequences of viral taxa, 3,446 bacterial genomes or scaffolds, 206 fungi related to human infection, and 140 parasites associated with human diseases. The depth and coverage of each species were calculated with the Soap Coverage software from the SOAP website (http://soap.genomics.org.cn/). The parameter values were normalized according the data size, which is 8 million reads for sputum and BAL, and 20 million reads for other samples. The detected species that existed in suspected background database or/and was also detected in negative control sample was filtered, if reaching the threshold $(18,19)$. The entire pipeline from the raw sequence data to the alignment to reference databases are provided in Figure S1.

\section{Sequencing data analysis}

To set up the threshold and minimize inaccurate assignments due to environmental contaminants, specimen collection and focal colonization, we processed 160 samples with each group collected from patients with non-inflammatory disease from Dec 2016 to Feb 2017, included the following: plasma $(n=50)$, sputum $(n=30)$, $\operatorname{CSF}(n=50)$, and BAL $(n=30)$. Analysis of the NGS results involved the following protocol (20-22): at least 3 reads were mapped to the pathogens whose relative abundances surpassed their threshold determined by the preliminary sequencing data. The preliminary data contained the relative abundance of microbes detected by NGS in healthy control samples; based on this data, we set up each microbe's threshold for further validation. Pathogens owned the highest absolute abundance in their genus; pathogens ranked top 10 for bacteria, viruses, and parasites and ranked top 20 for fungi and Mycobacterium tuberculosis in relative abundance after the previous two screening steps. After the prior analysis, if the detected pathogens were commonly reported infectious pathogens, they were be considered causative agents, while for noncommonly reported pathogens, NGS results were in accordance with the patient's clinical features, otherwise the detected reads were classified as non-pathogenic microbe sequences.

\section{Statistical analysis}

Sensitivity, specificity, positive predictive values (PPVs), negative predictive values (NPVs), and the associated 95\% confidence intervals (CIs) were calculated according to the definitions provided in Table S1. The proportions were compared using the Chi-squared test or Fisher's exact test, as appropriate. Test concordance was assessed using the kappa $(\kappa)$ statistic. $P$ values $<0.05$ were considered significant. Statistical analyses were conducted using SPSS Version 19.0 (IBM Corp., Armonk, NY, USA) and figures were rendered with GraphPad Prism 7.0 (GraphPad Software, San Diego, CA, USA).

\section{Results}

\section{General characteristics}

In the present study, a total of 141 patients were enrolled for eligibility and 108 patients were included (Figure 1). The baseline characteristics of the patients are listed in Table 1. Factors including gender, age, body temperature, C-reactive protein (CRP), erythrocyte sedimentation rate (ESR), procalcitonin (PCT), white blood cell (WBC), and the percentage of neutrophils were all equally distributed among three groups. Among the 108 patients, 36 were diagnosed with definite infections by both clinical and microbiological criteria (group I) and 41 patients were diagnosed with infection by clinical criteria only (group II). Thirty-one patients were ultimately diagnosed with non-infectious diseases (group III) including autoimmune encephalitis, malignant tumors, tsutsugamushi disease, drug fevers, and so on (Table 1). Seventeen (15.7\%) of the enrolled patients received antibiotic therapy and 39 patients $(36.1 \%)$ received symptomatic treatment prior to participation for less than 3 days. Among the 108 patients enrolled, 5 died during the study period ( 2 in group I and 3 in group II); death was attributable to infection in 4 cases.

\section{Diagnostic performance of NGS in clinically and microbiologically positive subjects}

The comparison of the diagnostic results of NGS with the CMT method for all 108 patients is shown in Table 2 and Figure 2A. NGS and CMT were concordant for 72 of 108 (66.7\%) patients ( $\kappa=0.386$; $95 \%$ CI: 0.248 to 0.525 ). For the culture method and NGS, the agreement rate was $60.2 \%$ ( $\kappa=0.289 ; 95 \%$ CI: 0.161 to 0.417 ).

In the present study, the diagnosis of 36 patients was 
Table 2 Comparison of positive results and agreement among next-generation sequencing, conventional microbiological tests and cultivation method in immunosuppressed patients with suspected infection

\begin{tabular}{lcccc}
\hline Group & NGS-positive ${ }^{\text {a }}$ & NGS-negative & Total no. & Kappa, agreement \\
\hline CMT-positive & 34 & 2 & 36 & $0.386,66.7 \%$ \\
CMT-negative & 34 & 38 & 72 & $0.289,60.2 \%$ \\
Culture-positive & 27 & 2 & 29 & 79 \\
Culture-negative & 41 & 38 & 108 & \\
Total no. & 68 & 40 & 2 & \\
\hline
\end{tabular}

a , positive: patients with a positive microbiological diagnosis. NGS, next-generation sequencing; CMT, conventional microbiological tests.

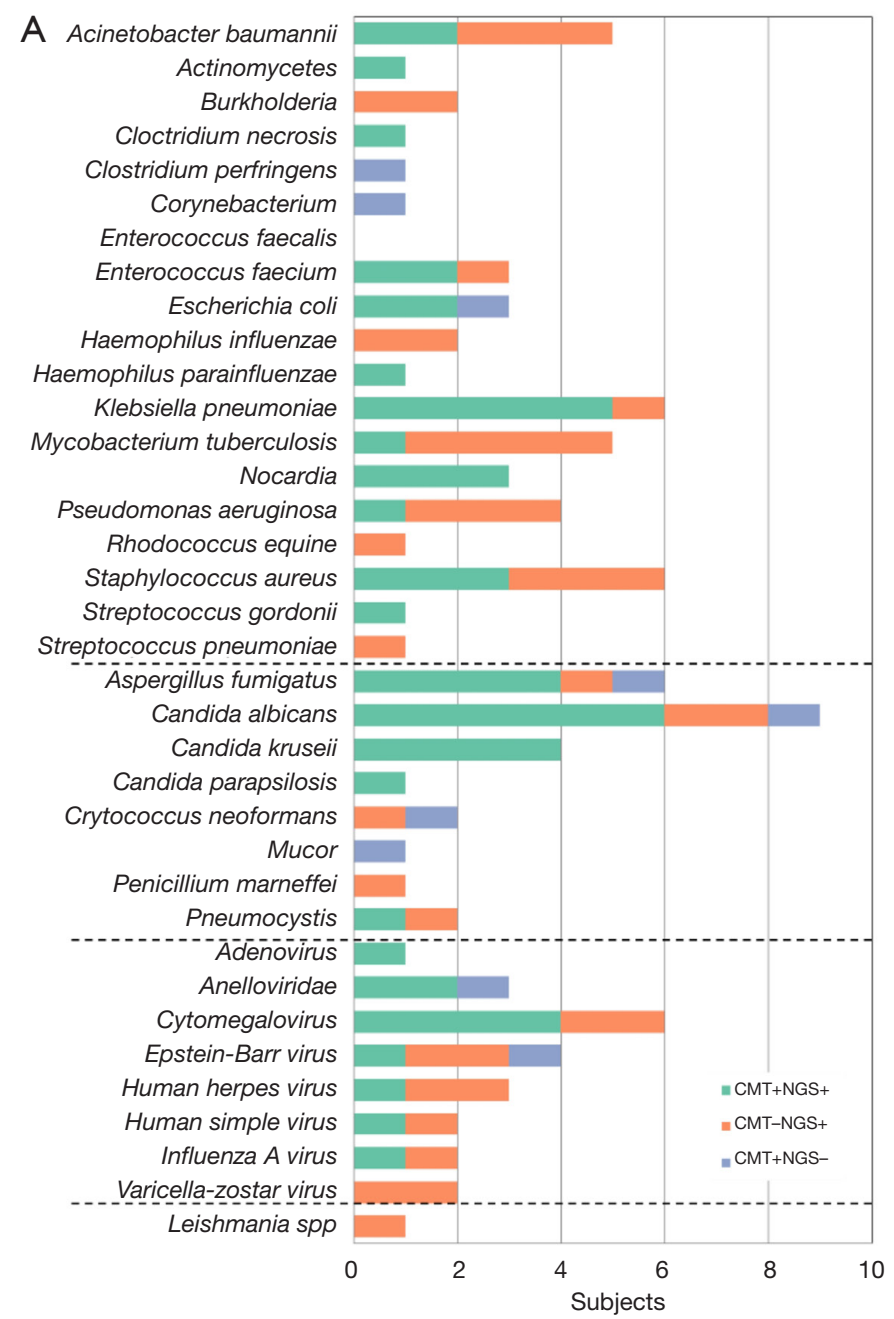

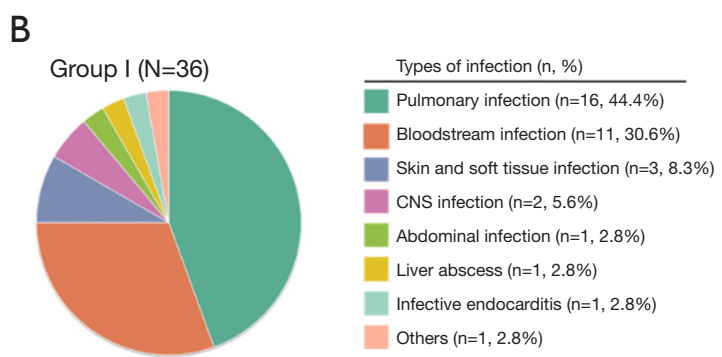

C
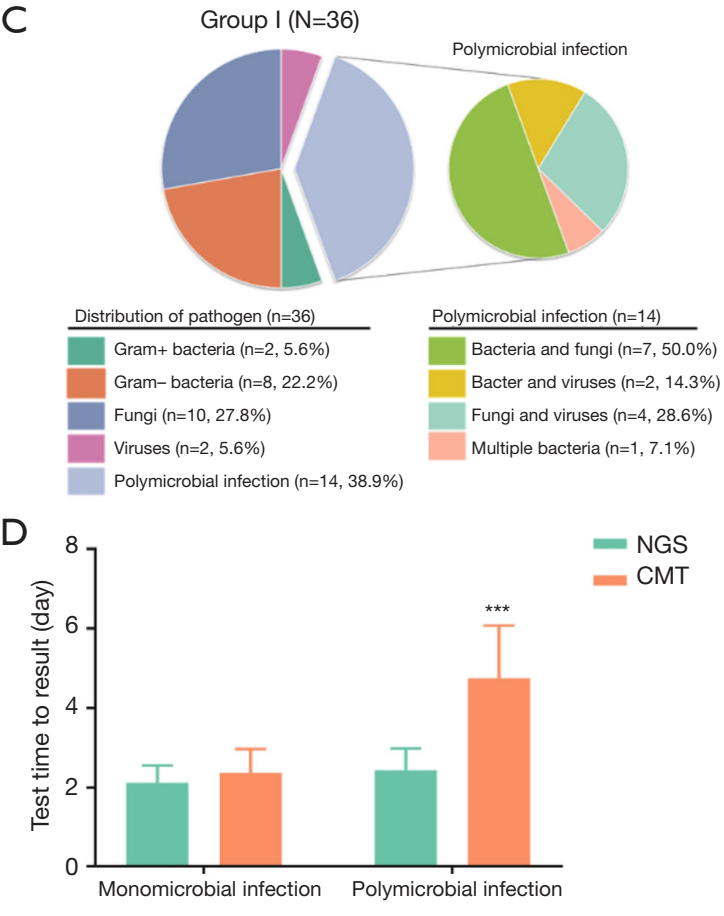

Figure 2 Distribution of pathogens identified in immunosuppressed patients using CMT versus NGS. (A) The figure showed the number of subjects in whom each microbe was detected. Green bars indicate microbes detected by CMT and also predicted as pathogens by NGS (CMT+NGS+). Orange bars indicate microbes detected by NGS only (CMT-NGS+). Blue bars indicate the number of cases with microbes detected only by CMT (CMT+NGS-); (B) distribution of types of infection was shown from patients in Group I; (C) distribution of pathogens was shown from patients in Group I. Polymicrobial infection accounted for 38.9\% among all the subjects and different kinds of polymicrobial infection were also shown in the right; (D) the diagnostic time required for NGS and CMT were compared in subjects with monomicrobial infection or polymicrobial infection in Group I. ${ }^{* * *}, \mathrm{P}<0.0001$ by Wilcoxon rank-sum test. 
Table 3 The detailed information of samples with conflicting results

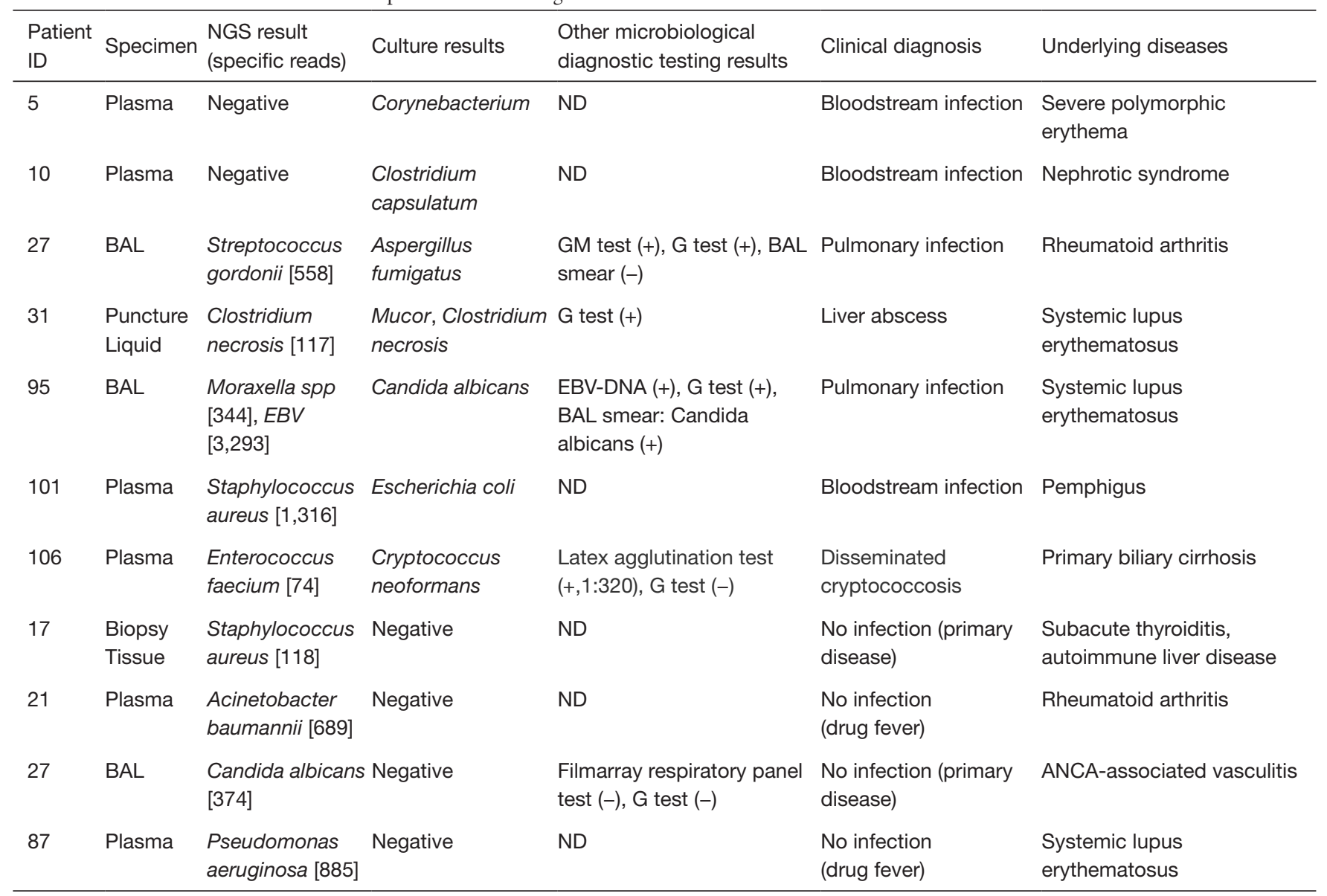

G test, (1,3)- $\beta$-D-glucan test. EBV, Epstein-Barr virus; BAL, bronchoalveolar lavage; GM test, Galactomannan test; ND, no data.

ultimately confirmed by both clinical and microbiological criteria (Figure 2B). According to the results, bacteria $(\mathrm{n}=11$, $30.6 \%)$ were the most common pathogens identified, followed by fungi $(n=9,25.0 \%)$, and virus $(n=2,5.6 \%)$; 14 patients were identified with more than one pathogen (Figure $2 A$ ). The top 3 causative pathogens identified were Klebsiella pneumonia $(\mathrm{n}=6)$, Candida albicans $(\mathrm{n}=4)$, and Aspergillus fumigatus $(\mathrm{n}=4)$. Among the 36 patients in Group I, NGS results for 29 subjects were consistent with CMT methods, with a coincidence rate of $80.6 \%$ (29/36). For 7 subjects with discordance results, NGS reported 2 false-negative cases, both of which were subjects with bloodstream infections (1 case with Corynebacterium and 1 case with Clostridium perfringens). Among the remaining 5 subjects, NGS detected only one pathogen in 1 case and different pathogens than those found via CMT in 4 cases (Table 3).
It is noteworthy that among the 36 subjects in group I, $14(38.9 \%)$ were diagnosed with polymicrobial infections, including 11 with pulmonary infections, 2 with bloodstream infections, and 1 with a liver abscess (Figure 2C). The diagnostic accuracy of NGS was $81.8 \%(18 / 22)$ in cases with polymicrobial infections, showing no statistical difference than that in cases with monomicrobial infections $(78.6 \%, 11 / 14)(\mathrm{P}>0.5)$. For samples from 14 patients with polymicrobial infections, multiple microbiological tests were performed to acquire final pathogenic diagnoses, requiring more time and cost than tests in subjects with monomicrobial infections (Table S2). However, NGS yielded consistent results from only a one-time test for 12 of the 14 subjects. We further analyzed the test time required to determine the pathogenic diagnosis (Table S2). For monomicrobial infections, the cycles required for NGS and CMT are not statistically different; for polymicrobial 
infections, however, NGS required significantly less time to identify the pathogens than CMT methods $(\mathrm{P}<0.0001)$ (Figure 2D).

\section{Diagnostic performance of NGS in patients with no evidence of infection}

For the 31 cases with no evidence of infection in Group III, NGS detected pathogens in specimens from 4 patients, as shown in Table 3. The microbes identified in the 4 cases by NGS were present at relatively low abundance (NGS semi-quantitative sequencing reads $<1,000)$. Overall, NGS showed a sensitivity of $80.6 \%(29 / 36)$ (95\% CI, $64.7 \%$ to $90.6 \%)$ and a specificity of $87.1 \%$ (27/31) (95\% CI, $70.5 \%$ to $95.5 \%$ ) when subjects in Group I were defined as patients and subjects in Group III were defined as controls. The NPV and PPV were $79.4 \%$ (95\% CI, $62.9 \%$ to $90.0 \%$ ) and $87.9 \%$ (95\% CI, $72.1 \%$ to $95.8 \%$ ), respectively.

\section{Identification of pathogen species in CMT negative samples by NGS}

In the present study, a total of 41 cases were diagnosed with infections based on clinical criteria only, with negative results from CMT. Among these 41 patients, NGS detected positive pathogens in 30 cases, including 11 cases with bacterial infections, 5 with fungal infections, 2 with viral infections, 1 with a protozoal infection, and 11 with polymicrobial infections.

In immunosuppressed patients with possible infections, developing targeted and precise antibiotic regimens without pathogenic evidence remains a substantial challenge. To assess the potential benefits of NGS in treatment decisionmaking, we retrospectively reviewed the antibiotic regimen received by the 41 patients in group II, as well as the clinical outcomes of their infections. According to whether antibiotic regimens were guided or modified by NGS results, the 41 patients were divided into four categories; the respective workflow of diagnosis and treatment is shown in Figure 3A. Clinical outcomes in terms of TSR on day 14 after admission were also evaluated by standard procedures (Table S1) and compared among different groups (A, B, C, and D).

A. Empiric antibiotic therapy was performed with negative NGS results $(\mathrm{n}=11)$. The overall TSR was $45.5 \%(5 / 11)$ and 2 patients died in this group;

B. The initial empirical antibiotic regimen was adjusted immediately upon access to NGS results $(\mathrm{n}=9)$ and the TSR was $77.8 \%(7 / 9)$. The treatment regimen of a representative case is shown in Figure 3B;

C. For 13 patients, empiric antibiotic therapy was initially performed. Modifications to initial therapy guided by NGS were applied if symptoms did not improve after 4-7 days of therapy. This strategy ultimately yielded a TSR of $84.6 \%(11 / 13)$. The treatment detail of a representative case is shown in Figure 3C;

D. Antibiotic therapy was performed without reference to NGS results throughout $(\mathrm{n}=8)$. Modification of antibiotic regimen was considered if symptoms did not improve 4-7 days after initial antibiotic therapy but without reference to NGS results. Among these 8 cases, the application of antibiotics was consistent with NGS results in 4 cases. The final TSR was $62.5 \%(5 / 8)$ after 14 days.

In general, with a treatment regimen based on or modified by NGS results $(B+C)$, the overall TSR was $81.8 \%(18 / 22)$. This was significantly higher than that observed in patients with treatment not based on NGS results $(52.6 \%, 10 / 19$; 2 died within 14 days; $\mathrm{A}+\mathrm{D})(\mathrm{P}<0.0001)$. These results indicated that NGS plays an important role in improving medical decision-making for optimized antibiotic treatment, especially in immunosuppressed patients with negative CMT results.

\section{Influence of immunosuppression on the diagnostic accuracy of NGS and CMT}

To establish whether the diagnostic performance of NGS was affected by degrees of immunosuppression, we calculated the association between cumulative doses of prednisone and the detection rate of NGS. Patients from groups I and II were combined as patients with infections. The cumulative steroid dose was chosen as an indicator combining the impact of both the daily dose of prednisone and the duration of therapy (3). According to the results, the positive rate of CMT decreased significantly when the cumulative dose was $>1,000 \mathrm{mg}$ (Figure $4 A$ ). However, the positive detection rates of NGS were not affected by the cumulative dose of prednisone used.

We also investigated the distribution of pathogens in patients with different degrees of immunosuppression. Analysis of the subgroups based on the proportion of pathogens is shown in Figure 4B. The proportion of polymicrobial infections was significantly higher as the cumulative dose of prednisone increased. The proportion of 
A

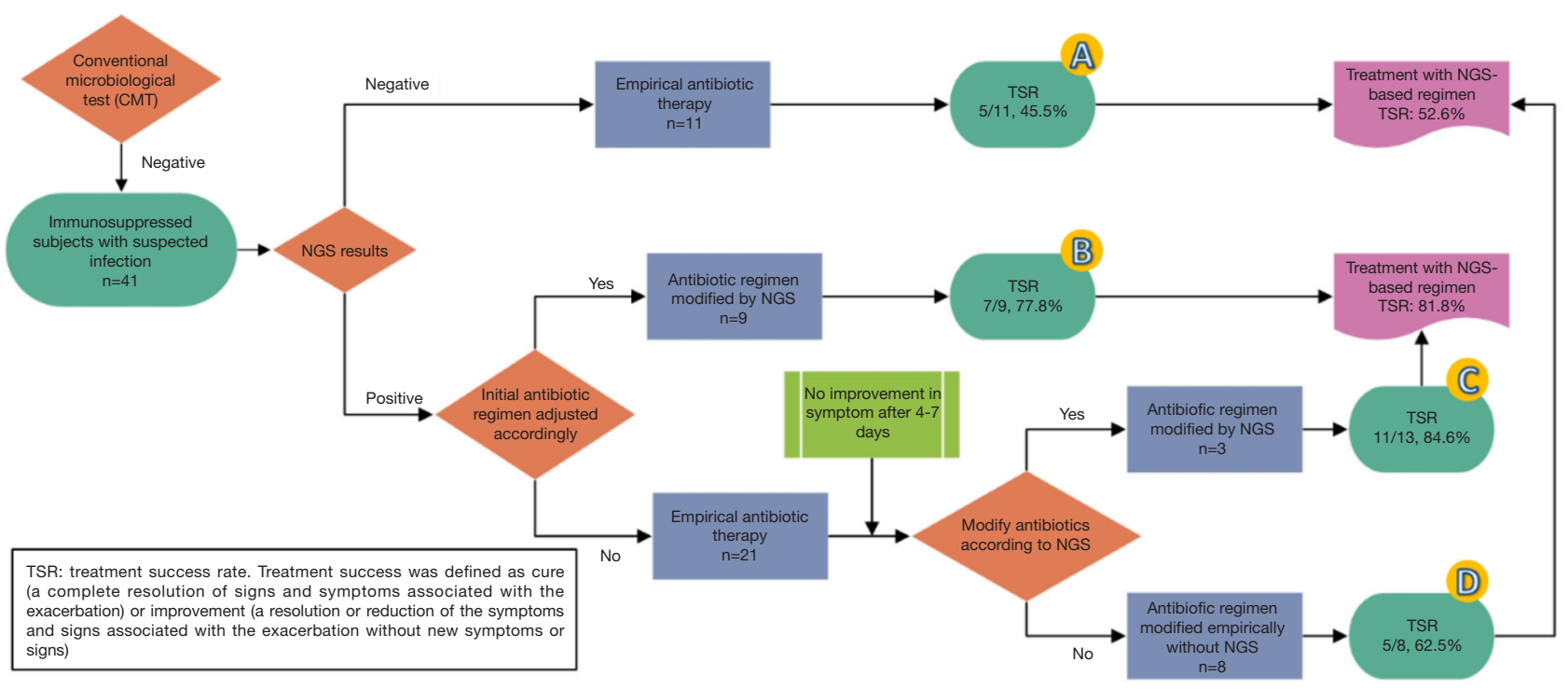

B

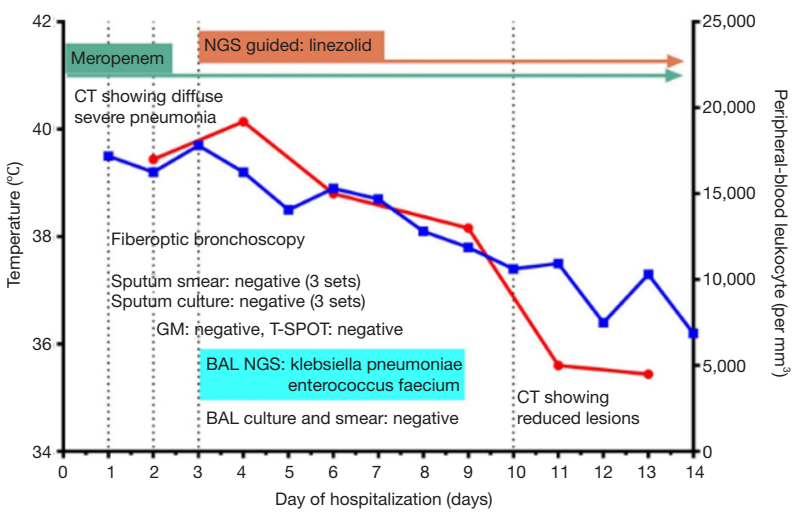

C

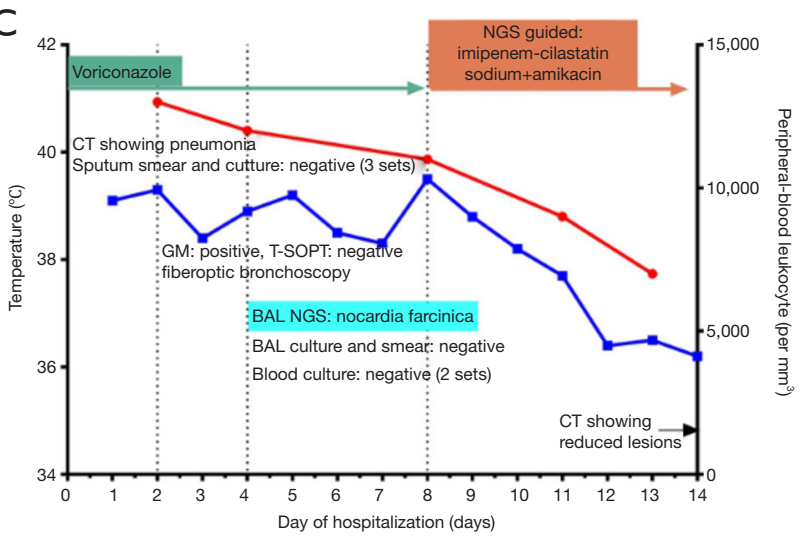

Figure 3 Diagnostic value of NGS in patients with negative CMT results. (A) Schematic representation of the different workflow showing the diagnosis and treatment processes guided by NGS results or not (workflow A, B, C and D in Figure 3A). Initial empirical antibiotic therapy was performed within $24 \mathrm{~h}$ after admission. Once they have initiated empirical antibiotics for fever, all patients were monitored closely for the response, adverse effects, emergence of secondary infections, and improvement of symptom. Modifications to the initial antibiotic regimen were considered for patients with persistent fever or no improvement in symptoms after 4-7 days of antibiotics. TSR was calculated on day 14 after admission to evaluate different treatment workflow; (B) representative case 1. An 83-year-old male patient developed a fever after 3 weeks of hormone therapy (methylprednisolone: $60 \mathrm{mg} / \mathrm{d}$ ) for polymorphic erythematous drug eruption. Voriconazole was given empirically but the symptom was not improved after 7 days of treatment. Then the antibiotic regimen was adjusted according to NGS results (Nocardia), which was obtained 4 days before. After 7-day's therapy the patient's symptoms were improved; (C) representative case 1. A 57-year-old female patient developed high fever and dyspnea. She has been taking long-term hormone therapy for systemic lupus erythematosus (methylprednisolone: $40 \mathrm{mg} / \mathrm{d}$ for 3 months). Initial empirical antibiotic regimen (meropenem) and then modified as soon as the NGS results were obtained (Acinetobacter baumannii and Enterococcus faecium). The patients' symptoms were finally improved within 14 days after admission. 

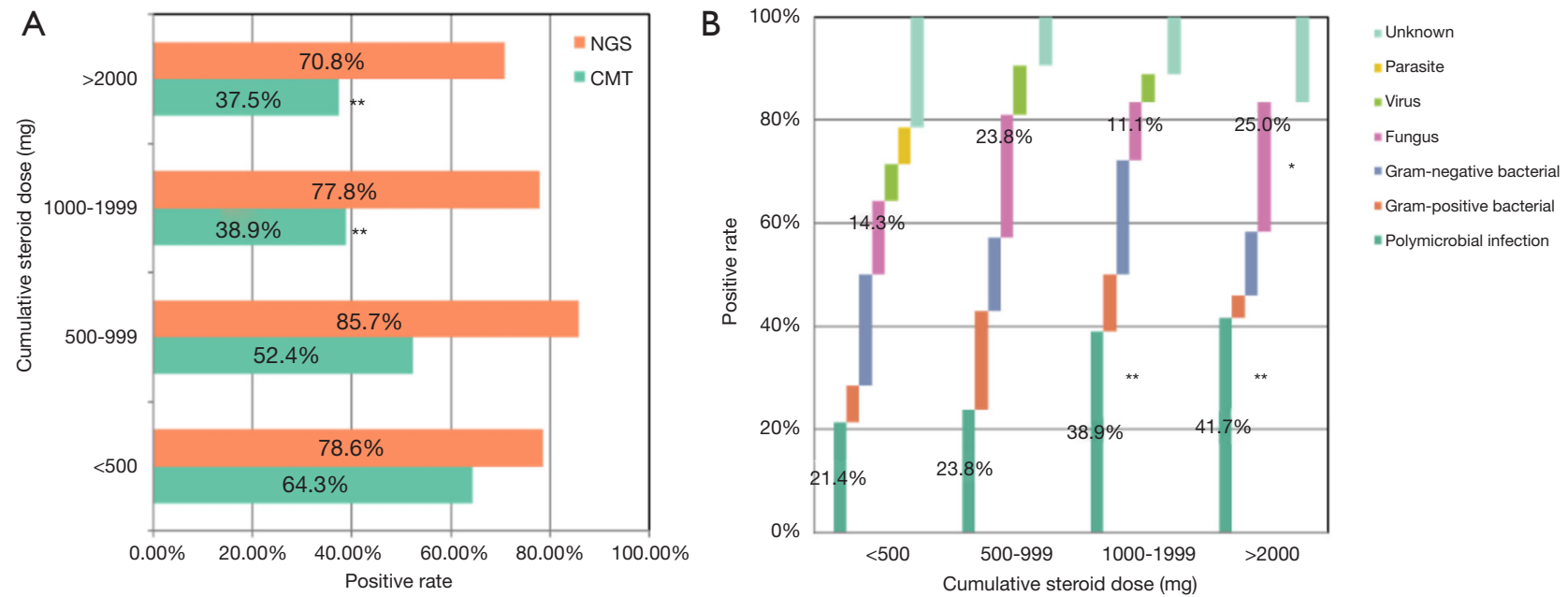

Figure 4 Influence of immunosuppression on the diagnostic accuracy of NGS and CMT. (A) The positive microbiological detection rate of NGS versus CMT in different subgroups divided by cumulative steroid dose; (B) the proportion of different kinds of pathogens detected by NGS and CMT in subgroups with different cumulative steroid dose. *, positive rate was significantly higher than that in subjects with cumulative steroid dose of $<500 \mathrm{mg}, \mathrm{P}<0.01 . \chi^{2}$ test. $^{* *}$, positive rate was significantly higher than that in subjects with cumulative steroid dose of $<500 \mathrm{mg}, \mathrm{P}<0.001 . \chi^{2}$ test.

fungal infections that could not be obtained by traditional culture methods also increased when the cumulative dose was $>2,000 \mathrm{mg}$ (Figure 4B). According to these results in immunosuppressed patients, the diagnostic accuracy of CMT can be influenced by the degree of immunosuppression, with an increased proportion of polymicrobial and fungal infections. However, NGS provides pathogenic diagnosis in immunosuppressed patients devoid of influence by these factors.

\section{Discussion}

In this prospective cohort study, we described the use of unbiased NGS for pathogenic diagnosis in immunosuppressed patients with suspected infections. The main result of this study is that NGS conveys substantial diagnostic potential in detecting infectious pathogens in samples from such immunosuppressed patients, which could be a valuable tool for complementing routine diagnostic methods, especially in cases with unknown etiology by CMT.

For immunocompromised patients with suspected infections, early and appropriate antibiotics remain the cornerstone for treatment in clinical practice (23-25). It is recommended that specimens from sites of suspected infection should be obtained and tested simultaneously as clinically indicated. Empiric antibiotic therapy should be performed initially according to the clinical manifestations. This initial antibiotic regimen should then be modified as determined by clinical and microbiological data if symptoms did not improve (26). In the present study, however, no pathogen was identified by standard clinical diagnostics in 41 patients with infections. A low detection rate by conventional methods such as culture was observed in several studies $(12,16,17)$, constituting a substantial obstacle for optimizing antibiotic therapy. In our study, we compared different clinical strategies for antibiotic regimens with or without reference to NGS; the results clearly demonstrated that NGS showed great potential to enhance antibiotic stewardship $(\mathrm{B}+\mathrm{C})$. The capacity for NGS to detect pathogens unidentifiable by CMT was highlighted in several cases in group II, facilitating medical decisionmaking and optimizing empiric antibiotic regimens. Therefore, although NGS results were not directly combined with CMT, which was used as gold standard for diagnosis in the present study, the diagnostic performance of NGS in Group II demonstrated that NGS could be major value for complementing the CMT, in particular in clinical or public health settings where routine diagnostics remain negative.

Moreover, in 31 patients with no evidence of infection, 19 were still treated with empirical antibiotics. This led to a waste of medical resources and unnecessary suffering as a result of several side effects. Due to the high NPV of NGS demonstrated by our study, NGS may be used to exclude fever as a sign of infection. The implementation of this rule-out strategy by NGS may reduce the excessive use of 
antibiotics as well as the days of antibiotic therapy in these patients. In further studies, we will enlarge the sample size to validate this diagnostic potential in prospective cohort study.

Several studies report that the proportion of polymicrobial infections in the immunosuppressed population will increase significantly, which is one of the most important features of immunocompromised hosts compared to ordinary patients $(27-31)$. This is due to the destruction of the host's immune function, making the host susceptible to various types of pathogens (1). Our results revealed that the diagnostic performance of CMT decreased for polymicrobial infections. The reason for these diagnostic defects is based on the characteristics of CMT. First, the testing mechanisms of single methods make it difficult to detect various kinds of pathogens. For instance, viruses and parasites cannot be detected by the culture method. PCR-based methods can only detect the presence of a limited range of organisms. The application of multiple detection methods greatly increases the time required for pathogen identification. In contrast, NGS provides a onestep solution, as it allows the sequencing and identification of all amplicons in one sample, thereby avoiding the problems encountered with the aforementioned assays and saving substantial time to diagnosis (32).

In the present study, certain cases existed wherein the NGS results were inconsistent with those of the clinical microbiological methods. Possible explanations include the difficulty of sequencing of the circulatory genomic DNA of intracellular bacteria (e.g., Mycobacterium tuberculosis and Bacterium burger). Fungi tend to harbor hard-to-break cell walls, which may restrain the extraction of pathogen DNA segments. RNA viruses such as Japanese $B$ encephalitis virus require reverse transcription before deep sequencing; therefore, the accuracy of the detection rate might be reduced. For the 31 cases with no evidence of infection in Group III, NGS detected pathogens in specimens from 4 patients, which were considered as falsepositive results. The pathogens obtained by NGS could not always lead to clinical manifestation $(4,8)$. The falsepositive results may due to factors including background pathogen DNA contamination during library preparation, low-quality reads from the samples, misannotated species, or contaminants from database entries $(20,33)$. Further evaluation and establishment of strict operating rules might still be necessary. We also used a negative control in every sequencing run to control for contamination and all samples in this study were treated according to the same protocols in the same laboratory. Non-pathogenic microbe sequences detected in our study may come from three possible sources: environmental contamination, reagent contamination, and errors occurring during sequencing and mapping (Table S3).

In the present study, we recruited patients receiving corticosteroid therapy for the evaluation of NGS. Corticosteroids are the most widely used chemotherapeutic agents with anti-inflammatory and immunosuppressive properties. Notably, different immunosuppressive treatments have different effects on host defenses and are associated with susceptibility to particular infections (1). The increased risk of infection could also be affected by the choice of different immunosuppressive agents, ways of drug administration, and underlying diseases $(1,34)$. Therefore, further evaluation is necessary to recruit immunosuppressive patients with different characteristics, such as those receiving treatment with neoplasia, autoimmune diseases, solid organ and stem cell transplants, to assess the diagnostic effect of NGS.

This study has certain limitations. First, our sample size is relatively small, and further studies need to recruit a larger cohort of immunosuppressed patients with different susceptibilities to pathogens. Second, given that NGS results may be easily influenced by many factors, the standards used in our study were generated using preliminary study data and must be thoroughly modified and tested prior to use by other centers. Third, sequencing costs remain a concern for NGS in clinical practice for most people including patients and insurers. In our study, all the samples were transferred to a centralized laboratory in another city for NGS testing, which could increase transportation costs and the turnaround time. For further study, we are committed to exploring ways to reduce costs, including steaming workflow, automating library preparation, and localization of the sequencing platform, which could benefit the cost-effectiveness of developing NGS as a diagnostic tool. Finally, a small number of patients in our study received treatment for less than 3 days before recruitment. Effective symptomatic and antibiotic treatment could affect the diagnostic performance of both NGS and CMT. Although NGS was proved to be less likely to be affected by prior antibiotic usage than cultures $(35,36)$, further validation of the NGS in real-world practice is still necessary. Other areas such as the impact of treatment on NGS measurement readings and whether NGS can be used as a method to assess treatment effectiveness also require further evaluation in our future studies. 


\section{Conclusions}

Our findings suggest that NGS holds great promise for the detection of potential pathogens in the clinical samples of immunosuppressed hosts receiving corticosteroid therapy with suspected infection. NGS stands to become a useful tool in first-line diagnostic setups for infection detection and control and will provide valuable information to optimize antibiotic therapy in immunosuppressed patients.

\section{Acknowledgments}

We thank the patients for cooperating with our investigation and acknowledge the professionalism and compassion demonstrated by all the healthcare workers involved in patients' care.

Funding: This research was funded by Key Technologies Research and Development Program for Infectious Diseases of China (2018ZX10305-409-001-003) and Shanghai Shenkang Medicine Developing Project (SHCD12017104).

\section{Footnote}

Conflicts of Interest: The authors have no conflicts of interest to declare.

Ethical Statement: The authors are accountable for all aspects of the work in ensuring that questions related to the accuracy or integrity of any part of the work are appropriately investigated and resolved. An ethical review application was validated by the Ethical Review Committee of Huashan Hospital, Fudan University, Shanghai, China. Written informed consent was obtained for each patient or their legal designees prior to participation.

Open Access Statement: This is an Open Access article distributed in accordance with the Creative Commons Attribution-NonCommercial-NoDerivs 4.0 International License (CC BY-NC-ND 4.0), which permits the noncommercial replication and distribution of the article with the strict proviso that no changes or edits are made and the original work is properly cited (including links to both the formal publication through the relevant DOI and the license). See: https://creativecommons.org/licenses/by-nc-nd/4.0/.

\section{References}

1. Dropulic LK, Lederman HM. Overview of Infections in the Immunocompromised Host. Microbiol Spectr 2016. doi:10.1128/microbiolspec.DMIH2-0026-2016.

2. Rhen T, Cidlowski JA. Antiinflammatory action of glucocorticoids--new mechanisms for old drugs. N Engl J Med 2005;353:1711-23.

3. Stuck AE, Minder CE, Frey FJ. Risk of infectious complications in patients taking glucocorticosteroids. Rev Infect Dis 1989;11:954-63.

4. Goldberg B, Sichtig H, Geyer C, et al. Making the Leap from Research Laboratory to Clinic: Challenges and Opportunities for Next-Generation Sequencing in Infectious Disease Diagnostics. MBio 2015;6:e1888-15.

5. Lecuit $M$, Eloit $M$. The diagnosis of infectious diseases by whole genome next generation sequencing: a new era is opening. Front Cell Infect Microbiol 2014;4:25.

6. Wickes BL, Wiederhold NP. Molecular diagnostics in medical mycology. Nat Commun 2018;9:5135.

7. C AH, Caya C, Papenburg J. Rapid and simple molecular tests for the detection of respiratory syncytial virus: a review. Expert Rev Mol Diagn 2018;18:617-29.

8. Allcock RJN, Jennison AV, Warrilow D. Towards a Universal Molecular Microbiological Test. J Clin Microbiol 2017;55:3175-82.

9. Wilson MR, Naccache SN, Samayoa E, et al. Actionable diagnosis of neuroleptospirosis by next-generation sequencing. N Engl J Med 2014;370:2408-17.

10. Li H, Gao H, Meng H, et al. Detection of Pulmonary Infectious Pathogens From Lung Biopsy Tissues by Metagenomic Next-Generation Sequencing. Front Cell Infect Microbiol 2018;8:205.

11. Hasman H, Saputra D, Sicheritz-Ponten T, et al. Rapid whole-genome sequencing for detection and characterization of microorganisms directly from clinical samples. J Clin Microbiol 2014;52:139-46.

12. Tarabichi M, Shohat N, Goswami K, et al. Can next generation sequencing play a role in detecting pathogens in synovial fluid? Bone Joint J 2018;100-b:127-33.

13. Langelier C, Kalantar KL, Moazed F, et al. Integrating host response and unbiased microbe detection for lower respiratory tract infection diagnosis in critically ill adults. Proc Natl Acad Sci U S A 2018;115:E12353-62.

14. Thorburn F, Bennett S, Modha S, et al. The use of next generation sequencing in the diagnosis and typing of respiratory infections. J Clin Virol 2015;69:96-100.

15. Moustafa A, Li W, Singh H, et al. Microbial metagenome of urinary tract infection. Sci Rep 2018;8:4333.

16. Salzberg SL, Breitwieser FP, Kumar A, et al. Nextgeneration sequencing in neuropathologic diagnosis of 
infections of the nervous system. Neurol Neuroimmunol Neuroinflamm 2016;3:e251.

17. Grumaz S, Stevens P, Grumaz C, et al. Next-generation sequencing diagnostics of bacteremia in septic patients. Genome Med 2016;8:73.

18. Li H, Durbin R. Fast and accurate short read alignment with Burrows-Wheeler transform. Bioinformatics 2009;25:1754-60.

19. Fehlmann T, Reinheimer S, Geng C, et al. cPAS-based sequencing on the BGISEQ-500 to explore small noncoding RNAs. Clin Epigenetics 2016;8:123.

20. Zhang HC, Ai JW, Cui P, et al. Incremental value of metagenomic next generation sequencing for the diagnosis of suspected focal infection in adults. J Infect 2019;79:419-25.

21. Ai JW, Zhang HC, Cui P, et al. Dynamic and direct pathogen load surveillance to monitor disease progression and therapeutic efficacy in central nervous system infection using a novel semi-quantitive sequencing platform. J Infect 2018;76:307-10.

22. Zhu YM, Ai JW, Xu B, et al. Rapid and precise diagnosis of disseminated T.marneffei infection assisted by highthroughput sequencing of multifarious specimens in a $\mathrm{HIV}$-negative patient: a case report. BMC Infect Dis 2018;18:379.

23. Cazzola M, Rogliani P, Aliberti S, et al. An update on the pharmacotherapeutic management of lower respiratory tract infections. Expert Opin Pharmacother 2017;18:973-88.

24. Dumford DM 3rd, Skalweit M. Antibiotic-Resistant Infections and Treatment Challenges in the Immunocompromised Host. Infect Dis Clin North Am 2016;30:465-89.

25. Robilotti E, Holubar M, Seo SK, et al. Feasibility and applicability of antimicrobial stewardship in immunocompromised patients. Curr Opin Infect Dis 2017;30:346-53.

26. Cunha CB, Opal SM. Antibiotic Stewardship: Strategies to Minimize Antibiotic Resistance While Maximizing

Cite this article as: Wang S, Ai J, Cui P, Zhu Y, Wu H, Zhang W. Diagnostic value and clinical application of nextgeneration sequencing for infections in immunosuppressed patients with corticosteroid therapy. Ann Transl Med 2020;8(5):227. doi: 10.21037/atm.2020.01.30
Antibiotic Effectiveness. Med Clin North Am 2018;102:831-43.

27. Brogden KA, Guthmiller JM, Taylor CE. Human polymicrobial infections. Lancet 2005;365:253-5.

28. Trifilio S, Zhou Z, Fong JL, et al. Polymicrobial bacterial or fungal infections: incidence, spectrum of infection, risk factors, and clinical outcomes from a large hematopoietic stem cell transplant center. Transpl Infect Dis 2015;17:267-74.

29. Rolston KV, Bodey GP, Safdar A. Polymicrobial infection in patients with cancer: an underappreciated and underreported entity. Clin Infect Dis 2007;45:228-33.

30. Martin-Loeches I, M JS, Vincent JL, et al. Increased incidence of co-infection in critically ill patients with influenza. Intensive Care Med 2017;43:48-58.

31. Tudesq JJ, Cartron G, Riviere S, et al. Clinical and microbiological characteristics of the infections in patients treated with rituximab for autoimmune and/or malignant hematological disorders. Autoimmun Rev 2018;17:115-24.

32. Su Z, Ning B, Fang H, et al. Next-generation sequencing and its applications in molecular diagnostics. Expert Rev Mol Diagn 2011;11:333-43.

33. Zhang HC, Ai JW, Cui P, et al. Incremental value of metagenomic next generation sequencing for the diagnosis of suspected focal infection in adults. J Infect 2019;79:419-25.

34. Luna G, Alping P, Burman J, et al. Infection Risks Among Patients With Multiple Sclerosis Treated With Fingolimod, Natalizumab, Rituximab, and Injectable Therapies. JAMA Neurol 2019. [Epub ahead of print].

35. Miao Q, Ma Y, Wang Q, et al. Microbiological Diagnostic Performance of Metagenomic Next-generation Sequencing When Applied to Clinical Practice. Clin Infect Dis 2018;67:S231-40.

36. Xing XW, Zhang JT, Ma YB, et al. Evaluation of NextGeneration Sequencing for the Diagnosis of Infections of the Central Nervous System Caused by the Neurotropic Herpes Viruses: A Pilot Study. Eur Neurol 2018;80:283-8. 
Table S1 Supplementary methods

\begin{tabular}{|c|c|c|}
\hline Study design & \multicolumn{2}{|r|}{ Randomized, single-center, controlled clinical trial in Huashan Hospital in Shanghai, China } \\
\hline \multirow[t]{8}{*}{ Participants } & \multicolumn{2}{|r|}{$\begin{array}{l}\text { Inclusion criteria: immunosuppressed patients } 18 \text { years of age or older with suspected of infection were assessed for } \\
\text { potential inclusion. Patients were eligible for enrolment if they were suffering from an immunosuppressive condition } \\
\text { and suspected of having an infectious disease requiring microbiological examination }\end{array}$} \\
\hline & \multicolumn{2}{|c|}{ Exclusion criteria: } \\
\hline & \multicolumn{2}{|c|}{ (I) People under 18 years old } \\
\hline & \multicolumn{2}{|c|}{ (II) Known pregnancy; } \\
\hline & \multicolumn{2}{|r|}{ (III) Patients treated with antibiotics during the previous 2 weeks } \\
\hline & \multicolumn{2}{|r|}{ (IV) Psychiatric disorders or other inability to give written informed consent, not being available for follow-up } \\
\hline & \multicolumn{2}{|r|}{ (V) Patients suffering from severe organ dysfunction } \\
\hline & \multicolumn{2}{|r|}{ Included in this analysis: 108 out of 132 randomized participants finished at follow-up } \\
\hline \multirow[t]{9}{*}{ Outcomes } & \multicolumn{2}{|c|}{ - Antibiotic use } \\
\hline & \multicolumn{2}{|r|}{$\begin{array}{l}\text { - Treatment success rate (TSR) on day } 14 \text { after admission: TSR was defined as cure (a complete resolution of signs } \\
\text { and symptoms associated with the exacerbation) or improvement (a resolution or reduction of the symptoms and } \\
\text { signs associated with the exacerbation without new symptoms or signs) }\end{array}$} \\
\hline & (l) & Cure defined as resolution of clinical, laboratory, and radiographic signs of infection \\
\hline & & $\begin{array}{l}\text { Improvement was defined as reduction of clinical signs and symptoms, improvement of laboratory findings, } \\
\text { and reduction of the number or intensity of radiographic signs of infection }\end{array}$ \\
\hline & (III) & 1) Treatment success represented the sum of the rates for cure and improvement \\
\hline & & $\begin{array}{l}\text { Treatment failure included death, recurrence, relapse, or persistence of clinical, laboratory, and radiologic } \\
\text { signs of CAP and participants lost to follow-up }\end{array}$ \\
\hline & \multicolumn{2}{|c|}{ - Mortality } \\
\hline & \multicolumn{2}{|c|}{ - Duration of antibiotic therapy } \\
\hline & \multicolumn{2}{|c|}{ - Length of hospitalization } \\
\hline \multirow{14}{*}{$\begin{array}{l}\text { Conventional } \\
\text { Microbiological } \\
\text { test }(\mathrm{CMT})\end{array}$} & \multicolumn{2}{|c|}{ (I) Direct examination for fungal diagnosis } \\
\hline & \multicolumn{2}{|c|}{ (II) Blood culture } \\
\hline & \multicolumn{2}{|c|}{ (III) Bacterial and fungal stains and cultures } \\
\hline & \multicolumn{2}{|c|}{ (IV) AFB stains and cultures } \\
\hline & \multicolumn{2}{|c|}{ (V) Xpert MTB/RIF, Xpert MTB/RIF Ultra } \\
\hline & \multicolumn{2}{|c|}{ (VI) Filmarray respiratory Panel } \\
\hline & \multicolumn{2}{|r|}{ (VII) Serological tests for Epstein-Barr virus (EBV), cytomegalovirus (CMV) and influenza A/B } \\
\hline & \multicolumn{2}{|c|}{ (VIII) CMV and EBV DNA test } \\
\hline & \multicolumn{2}{|c|}{ (IX) Serum (1,3)- $\beta$-D-glucan test (G test) } \\
\hline & \multicolumn{2}{|c|}{ (X) Serum galactomannan test (GM test) } \\
\hline & \multicolumn{2}{|c|}{ (XI) T-SPOT TB test } \\
\hline & \multicolumn{2}{|c|}{ (XII) Latex agglutination test } \\
\hline & \multicolumn{2}{|c|}{ (XIII) 16s rRNA gene sequencing } \\
\hline & $(\mathrm{XIV})$ & India ink method for Cryptococcus neoformans \\
\hline
\end{tabular}

Criteria of The diagnosis of different types of infection was made by two independent clinicians, retrospectively reviewed each Infection patient's medical record including clinical, microbiological, and treatment outcome information, to determine whether they met the definition of different kinds of infections with respect to microbiologic and/or clinical criteria according to:

1) Centers for Disease Control and Prevention. (2019). CDC/NHSN Surveillance Definitions for Specific Types of Infections. Surveillance Definitions, (17), 1-29

2) Chen haozhu, Lin guowei, et al. Practice of Internal Medicine: $15^{\text {th }}$ Edition. People's Medical Publishing House Co., LTD., China, 2017

Statistical $\quad 2 \times 2$ contingency tables were derived to determine sensitivity, specificity, positive predictive value (PPV), negative analysis predictive value (NPV). All statistics have reported as absolute values with their $95 \%$ confidence interval $(95 \% \mathrm{Cl})$. Sensitivity and specificity were calculated on the basis of the formulas TP (true positive)/TP + FN (false negative) and TN (true negative)/TN + FP (false positive), respectively. PPV is expressed by the TP/TP + FP ratio, while NPV from the $\mathrm{TN} / \mathrm{TN}+\mathrm{FN}$ 
Table S2 Test time to results for NGS and CMT in Group I

\begin{tabular}{|c|c|c|c|c|c|c|c|}
\hline \multirow{2}{*}{$\begin{array}{l}\text { Patient } \\
\text { ID }\end{array}$} & \multirow{2}{*}{$\begin{array}{l}\text { Polymicrobial } \\
\text { infection }\end{array}$} & \multicolumn{2}{|c|}{ Test time to results (days) } & \multirow{2}{*}{ CMT } & \multirow{2}{*}{ Results } & \multirow{2}{*}{ Specimen } & \multirow{2}{*}{ Clinical diagnosis } \\
\hline & & NGS & CMT & & & & \\
\hline 1 & No & 2 & 1.5 & Smear and culture & Aspergillus & BAL & Pulmonary infection \\
\hline 2 & No & 2.5 & 2 & Blood culture & Candida albicans & Plasma & Bloodstream infection \\
\hline 3 & No & 2 & 2.5 & Blood culture & Escherichia coli & Plasma & Bloodstream infection \\
\hline 4 & No & 2 & 2 & Blood culture & Corynebacterium & Plasma & Bloodstream infection \\
\hline 5 & Yes & 2.5 & 2 & Smear and culture & Aspergillus, Nocardia & BAL & Pulmonary infection \\
\hline 6 & Yes & 3 & 2 & Smear and culture, Filmarray & Candida albicans, HSV 1 & BAL & Pulmonary infection \\
\hline 7 & No & 2 & 2.5 & Blood culture & Staphylococcus aureus & Plasma & Bloodstream infection \\
\hline 8 & Yes & 3 & 5 & Smear and culture & Aspergillus, Haemophilus parainfluenzae & BAL & Pulmonary infection \\
\hline 9 & No & 2 & 1.5 & Culture & Pseudomonas aeruginosa & Skin secretion & Skin and soft tissue infection \\
\hline 10 & No & 1.5 & 2 & Xpert & Mycabacterium tuberculosis & Sputum & Pulmonary infection \\
\hline 11 & No & 2 & 2 & Blood culture & Clostridium capsulatum & Plasma & Bloodstream infection \\
\hline 12 & Yes & 2.5 & 3.5 & Smear and culture & Candida albicans, Staphylococcus aureus, Klebsiella pneumoniae & Sputum & Pulmonary infection \\
\hline 13 & Yes & 2 & 4.5 & Smear and culture & Aspergillus, streptococcus gordonii & BAL & Pulmonary infection \\
\hline 14 & No & 3 & 3 & Blood culture & Candida albicans & Plasma & Hepatospenic candidiasis \\
\hline 15 & No & 1.5 & 2.5 & Blood culture & Klebsiella pneumoniae & Plasma & Bloodstream infection \\
\hline 16 & Yes & 3 & 5 & Smear and culture, Filmarray & Candida krusei, Influenza A & BAL & Pulmonary infection \\
\hline 17 & No & 2 & 3 & Culture & Candida albicans & Ascitic fluid & Peritonitis \\
\hline 18 & No & 2.5 & 2 & Blood culture & Candida krusei & Plasma & Bloodstream infection \\
\hline 19 & No & 2 & 1.5 & Blood culture & Candida krusei & Plasma & Bloodstream infection \\
\hline 20 & No & 2 & 2 & Culture & Candida krusei & BAL & Pulmonary infection \\
\hline 21 & Yes & 3.5 & 4 & Culture & Candida albicans, Clostridium necrosis & Puncture fluid & Liver abscess \\
\hline 22 & No & 2.5 & 2 & Smear and culture & Candida albicans & Sputum & Pulmonary infection \\
\hline 23 & No & 2.5 & 3 & Smear and culture & Aspergillus & Puncture fluid & Skin and soft tissue infection \\
\hline 24 & Yes & 2.5 & 3.5 & Smear and culture, EBV-DNA, EBV IgM & Candida albicans, EBV & BAL & Pulmonary infection \\
\hline 25 & No & 1.5 & 2 & Blood culture & Candida albicans & Puncture fluid & Kidney abscess \\
\hline 26 & No & 3 & 2.5 & Blood culture & Escherichia coli & Plasma & Bloodstream infection \\
\hline 27 & Yes & 2 & 2 & Culture & Klebsiella pneumoniae, Acinetobacter baumannii & BAL & Pulmonary infection \\
\hline 28 & Yes & 2 & 5 & Smear and culture & Aspergillus, Nocardia & BAL & Pulmonary infection \\
\hline 29 & Yes & 1.5 & 7 & Smear and culture & Candida albicans, Klebsiella pneumoniae & BAL & Pulmonary infection \\
\hline 30 & No & 2 & 3 & Culture & Klebsiella pneumoniae & Drainage fluid & Skin and soft tissue infection \\
\hline 31 & Yes & 2 & 4.5 & Blood culture, CMV-DNA, CMV IgM & Staphylococcus aureus, CMV & Plasma & Bloodstream infection \\
\hline 32 & No & 1.5 & 3.5 & Blood culture & Escherichia coli & Plasma & Bloodstream infection \\
\hline 33 & No & 2 & 2.5 & Blood culture & Cryptococcus neoformans & Plasma & Disseminated cryptococcosis \\
\hline 34 & Yes & 2.5 & 5.5 & Blood culture & Candida albicans, Acinetobacter baumannii & Plasma & Bloodstream infection \\
\hline 35 & No & 2.5 & 3.5 & Blood culture & Nocardia & Puncture fluid & Liver abscess \\
\hline 36 & Yes & 2 & 7 & Smear and culture & Enterococcus faecium, Adenovirus & BAL & Pulmonary infection \\
\hline
\end{tabular}




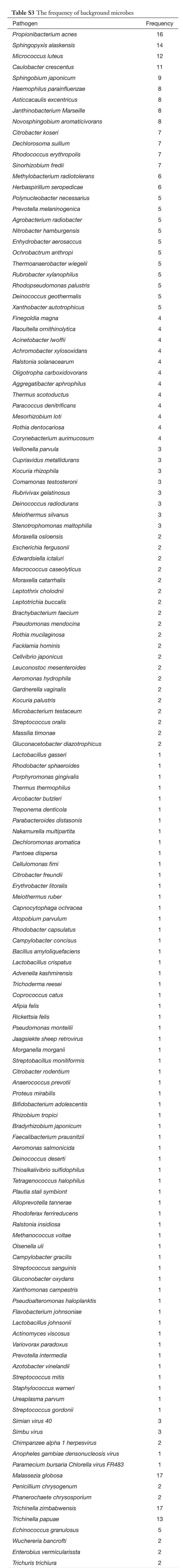




\begin{tabular}{|c|c|c|c|c|c|}
\hline & Reads No. & Reads percent, \% & Time, min & Tool & Database \\
\hline $\begin{array}{l}\text { Raw reads } \\
\text { fastq files }\end{array}$ & $\begin{array}{c}20636180 \\
\pm \\
2^{*} 12713904\end{array}$ & 100 & 0.0 & NA & NA \\
\hline \multicolumn{6}{|l|}{$\nabla$} \\
\hline Quality filter & $\begin{array}{c}218020 \\
\pm \\
2 * 140391\end{array}$ & $1.1^{\mathrm{a}}$ & 15.0 & $\begin{array}{l}\text { made-in-house } \\
\text { program }\end{array}$ & NA \\
\hline \multicolumn{6}{|l|}{$\nabla$} \\
\hline $\begin{array}{l}\text { Clean reads } \\
\text { fastq files }\end{array}$ & $\begin{array}{c}20423604 \\
\pm \\
2^{\star} 12579625\end{array}$ & 100 & 0.0 & NA & NA \\
\hline $\begin{array}{l}\text { Map to human } \\
\text { reference }\end{array}$ & $\begin{array}{c}1931223 \\
\pm \\
2 * 1303482\end{array}$ & $94.6^{\mathrm{b}}$ & 8.0 & BWA & $\mathrm{Hg} 38 / \mathrm{YH}$ \\
\hline $\begin{array}{l}\text { Alignment to bacterial// } \\
\text { virus/fungal/parasite DB }\end{array}$ & - & $1^{\mathrm{b}}$ & 5.0 & BWA/samtools & $\begin{array}{l}\text { PMseq genome } \\
\text { Database }\end{array}$ \\
\hline $\begin{array}{l}\text { Taxonomic and } \\
\text { reporting }\end{array}$ & - & - & 0.5 & $\begin{array}{l}\text { made-in-house } \\
\text { program }\end{array}$ & $\begin{array}{c}\text { PMseq Taxonomy } \\
\text { Database }\end{array}$ \\
\hline
\end{tabular}

a, of raw reads; b, of clean reads.

Figure S1 Bioinformation pipeline for sequencing data analysis. 